\title{
Detecting (Unusual) Events in Urban Areas using Bike-Sharing Data
}

\author{
Alex Lam \\ lamalex449@gmail.com \\ Rowan University \\ Glassboro, New Jersey
}

\author{
Matthew Schofield \\ schofieldmatthew86@gmail.com \\ Rowan University \\ Glassboro, New Jersey
}

\author{
Shen-Shyang Ho \\ hos@rowan.edu \\ Rowan University \\ Glassboro, New Jersey
}

\begin{abstract}
Social media, traffic sensors, GPS trajectories, and location-based social network data provide diverse spatio-temporal information sources that help to detect and analysis spatio-temporal events. Nowadays, bike sharing systems are active all over the world in major cities, and collecting a large amount of data regarding trips taken by users and status of the stations. Through analysis of the data aggregated by bike sharing systems, one can gain an understanding of crowd/commuter movements and behaviors. However, no one has used only the bike sharing data for generic event detection.

In this paper, we propose a clustering-based detection method to identify spatiotemporal events that deviate from normal or regular everyday life using publicly available bike sharing data. In particular, we apply spectral clustering on bike station and bike flow data as evolving graphs and monitor changes of the bike share network (edge/node values) over time. Our proposed method decides whether a cluster is expected or anomalous (unusual). When a cluster is anomalous, there is an unusual event occurring at that time instance. Preliminary results on 6-months of data from Philadelphia and Washington DC are used to show the feasibility of our proposed method. In particular, our preliminary results show that some signatures of local (and less prominent) events (e.g., university events/activities in an urban area) can show up when bike sharing data is utilized for generic event detection.
\end{abstract}

\section{CCS CONCEPTS}

- Computing methodologies $\rightarrow$ Anomaly detection; • Information systems $\rightarrow$ Spatial-temporal systems; Data mining.

\section{KEYWORDS}

bike sharing, spectral clustering, event detection, anomaly detection, graph analysis

\section{ACM Reference Format:}

Alex Lam, Matthew Schofield, and Shen-Shyang Ho. 2019. Detecting (Unusual) Events in Urban Areas using Bike-Sharing Data. In 3rd ACM SIGSPATIAL International Workshop on Analytics for Local Events and News (LENS'19), November 5, 2019, Chicago, IL, USA. ACM, New York, NY, USA, 7 pages. https://doi.org/10.1145/3356473.3365190

Permission to make digital or hard copies of all or part of this work for personal or classroom use is granted without fee provided that copies are not made or distributed for profit or commercial advantage and that copies bear this notice and the full citation on the first page. Copyrights for components of this work owned by others than ACM must be honored. Abstracting with credit is permitted. To copy otherwise, or republish, to post on servers or to redistribute to lists, requires prior specific permission and/or a fee. Request permissions from permissions@acm.org.

LENS'19, November 5, 2019, Chicago, IL, USA

(C) 2019 Association for Computing Machinery.

ACM ISBN 978-1-4503-6958-9/19/11 ..\$15.00

https://doi.org/10.1145/3356473.3365190

\section{INTRODUCTION}

Many different types of data such as twitter data [11, 20, 22], mobility and trajectory data [17, 21,24], mobile phone call detail records $[5,6]$, general social media data [25] can be used for spatial or spatiotemporal event detection and analysis. Recently, there are new interests in how bike sharing data can be used for specific spatiotemporal event detection such as vehicle illegal parking events [7] and also to use with other data sources for anomaly detection in an urban area [24].

Bike sharing systems have been in existence for almost sixty years, but in the past two decades they have risen in popularity [10]. Today these bike sharing systems are active all over the world in major cities, and collect a large amount of data regarding trips taken by users and status of the stations. Through analysis of the data aggregated by bike sharing systems, we can gain an understanding of crowd/commuter movements and behaviors [16, 27]. This will also allow cities to plan in advance for crowd movements and events that attract the attention and movement of citizens. Cities can use insights to better plan for events and their entailing crowd movements. Such information can aid in the proper planning and allocation of resources, such as security forces, public transportation and event advertising. Improving the flow and safety of crowd movement.

Additionally, analysis of this data historically can be paired with real time station reported data to identify anomalous behavior. A rapid influx to an area could indicate a spontaneous event, perhaps a celebration or riot, that warrants additional resources to be diverted to that area to maintain safety. On the contrary a rapid anomalous out flow from an area could indicate there is a dangerous event warranting immediate response. Such detection can provide early warnings that an event may be occurring allowing for faster response times, early warnings to dangerous events such as terrorism could prove life saving.

In this paper, we propose a clustering-based detection method to identify spatiotemporal events that deviate from normal or regular everyday life using publicly available bike sharing data. In particular, we apply spectral clustering on bike station and bike flow data as evolving graphs and monitor changes of the bike share network (edge/node values) over time. Our proposed method decides whether a cluster is expected or anomalous. When a cluster is anomalous, there is an interesting event occurs at that time instance. We apply our proposed method to two major bike sharing systems in the United States of America; Washington, DC and Philadelphia, PA.

While Zheng et al. [26] proposed using bike sharing data together with 311 complaints data, taxicab data, points of interest data, and road network data for anomaly detection, to collectively identify 
anomaly in an urban area (e.g., New York City), anomaly signatures that only show up in one dataset may be ignored. Also, He et al. [7] used bike sharing data for the specific tasks of detecting vehicle illegal parking. To the best of the authors' knowledge, there is no previous work on whether one can detect generic local events using ONLY bike sharing system data. Here, our preliminary results show that some signatures of local (and less prominent) events (e.g., school events/activities in an urban area) can show up when bike sharing data is utilized for event detection.

The layout of the remainder of this paper is as follows: Section 2 describes briefly some related work. Section 3 provides the methodology and history of the algorithm, Section 4 provides the background on the data used in this paper. Section 5 provides the results and discusses the events that have been flagged. Section 6 discusses and describes some of the issues related the our detection task. Section 7 provides conclusions and future work.

\section{RELATED WORK}

Social media, traffic sensors, GPS trajectories, and location-based social network data provide diverse spatio-temporal information sources that help to detect and analysis spatio-temporal events. For example, many techniques have been proposed for event detection from twitter data [2] (and reference therein). Recently, Suma et al [18] proposed an automated process that uses Twitter data to discover congestion and events in London. To detect traffic events such as congestion, accidents, delays, and traffic jams, wireless sensor network data have been widely used [14]. Social events can be detected using taxi traces [24]. Traffic congestion and accidents can also be detected from vehicle GPS traces [4]. Aoki et al. [1] proposed using bus GPS traces to predict events such as traffic congestion before the events become more severe. Dong et al. [6] proposed using mobile phone call data record to predict unusual crowded events.

They are many challenges in bike-sharing systems. For example, there are issues related to best placement of bikes to optimize usage, handling rush hour usage, rebalancing overnight to prepare for next day usage, etc. Data driven approaches using bike sharing system data have been proposed to solve these problems using publicly available system data [15]. Earlier work rely on assumptions when the bike-sharing systems did not release their trip data [13]. However, the bike sharing system data have becoming publicly available. Hence, most proposed approaches are now data driven. Gan et al. [9] proposed new approaches to position different bike stations [9]. Yang et al. [23] propose a new mobility modeling and prediction method which can help to solve the bike rebalancing problem. Recently, graph convolutional neural network approach has been proposed to predict bike demand at individual stations [12].

\section{METHODOLOGY}

In Section 3.1, we describe how the bike sharing data are transformed to graphs. Section 3.2 describes how spectral clustering is used to partition the bike stations into clustering from the graph representation derived from the bike sharing data. We present and discuss the event detection algorithm in Section 3.3.

\subsection{Representing Network of Bike Stations as Evolving Graphs}

In this paper, we represent the bike sharing systems in cities as evolving graphs. The nodes of these graphs being bicycle stations and edges being the number of bikes that travel between the two connected stations during a given time interval. The evolving graphs are represented by adjacency matrices that correspond to individual days. In order to use spectral clustering [8] to partition the graphs, we create an undirected matrix that corresponds to each day. We use spectral clustering as its use of adjacency matrices naturally lends itself to work with graphs.

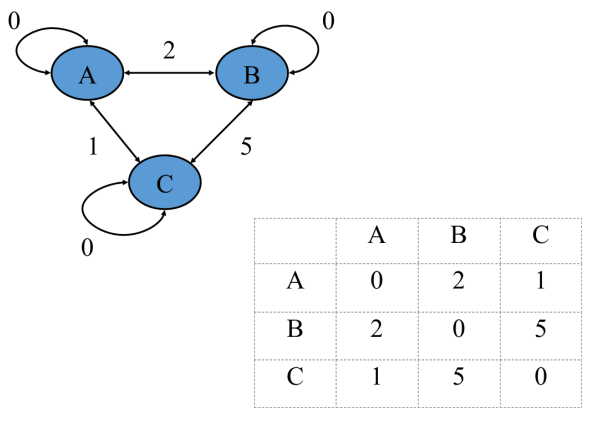

Figure 1: Visual Representation of 3 bike stations (A, B, C) as an undirected graph with the edge value represent the movement of bikes between 2 stations and the corresponding matrix representation. Note that in this example, no bike returns to its own station.

We then calculate the affinity matrix that corresponds to our generated matrix. For our proposed approach, we use the number of trips between stations as our partitioning variable. The affinity matrix represents the amount of activity two stations have to each other. Figure 1 shows an example of the graphs of 3 bike stations and the corresponding undirected matrix representation.

A $s \times s$ affinity matrix is generated to represent a single day's activity such that $s$ is the number of bike stations. We will create as many matrices as allowed within our data set. Each row vector in these matrices represents a single station's relation to every other station within the system. Our goal is to figure out which stations have similar interactions and assume based on those interactions, the real world relation of these stations. We hypothesize that the relations of different areas of a city are expressed in relations of their bike stations, and that unexpected (flow) changes in the bike stations expressed relation is indicative of an unexpected crowd movement in the broader city.

\subsection{Spectral Clustering to Partition related Bike Stations}

Spectral clustering has been widely used in recent years especially on graph represented as a matrix [19]. It was popularized by Shi and Malik [8] with their work on image segmentation. Primarily, spectral clustering relies on the Laplacian matrix of a graph and determining the eigenvectors in order to create partitions. There 
are many research work on spectral clustering algorithm for large scale clustering [3]

Once the matrices described in Section 3.1 are created and properly formatted, we apply spectral clustering to determine how we can partition the bike stations. We apply spectral clustering with varying number of allowed clusters. Our research currently focuses on setting the maximum to two clusters and we intend to later expand upon this. The reasoning of why we use two clusters is because the matrices are primarily sparse in nature. Because when there are too few activities to track, the partitioning becomes purely spatial. Moreover, with two clusters, there will be one dominating cluster representing the normal behavior and a smaller cluster that may contain interesting events. Note that when the bike stations in the smaller cluster are projected back to the spatial dimension, they may not be close to each other.

\subsection{Event Detection Algorithm}

Next, we calculate the spatial (latitude and longitude) center of every cluster using an unweighted average of each cluster member station's spatial location. Then, we calculate the center of each previously calculated clusters' midpoints. We track the anomaly score defined by the distance between the city's center and the clusters' average midpoint as it evolves over the given time interval.

The tracking of the midpoints' differences yields a 1-D timeseries, with data at each time increment used to produce a matrix. An event is detected based on a threshold value assuming a Gaussian distribution for the midpoints' differences. The threshold is chosen based on standard deviation according to the user-defined confidence. For example, a threshold chosen at 2 times the standard deviation will have at least $95 \%$ confidence that the detected event is a significant event and a threshold chosen at 3 times the standard deviation will have at least $99.7 \%$ confidence.

Algorithm 1 summarizes the steps taken to detect an event. In step 1, a spectral clustering algorithm, Spec_Clust is used to cluster the bike station given the generated affinity matrix, $M$ and the pre-defined number of clusters, $n c$. $C$ consists of $n c$ partitions of the bike stations using spectral clustering. Step 2 to 4 compute the spatial center, $m p_{i}$, of each cluster using $c_{i}$ which consists of all the bike stations in a cluster of index $i$. Step 5 computes the center $c e n$ of all the cluster centers, $m p_{i}, i=1, \cdots, n c$. Step 6 computes the anomaly score, $A S$, which corresponds to the Euclidean norm (distance) between the city center and the center of the cluster centers. Step 7 to 9 is the thresholding detection procedure using threshold value $T$ described above.

\section{DATA}

We tested our algorithm on two different cities major bike sharing systems in the United States of America: Washington, DC and Philadelphia, PA. The first system we selected was Philadelphia's Indego bike system first released in 2015. Indego BikeShare has over 130 available stations and over 1,000 bikes $^{1}$. Figure 2 shows the station locations for Philadelphia's Indego bike sharing system. The second system we have selected is Washington DC's Capital bike sharing system ${ }^{2}$. It has over 500 rental stations and has over 4,300

\footnotetext{
${ }^{1}$ https://www.rideindego.com/about/data/

${ }^{2}$ https://www.capitalbikeshare.com/system-data
}
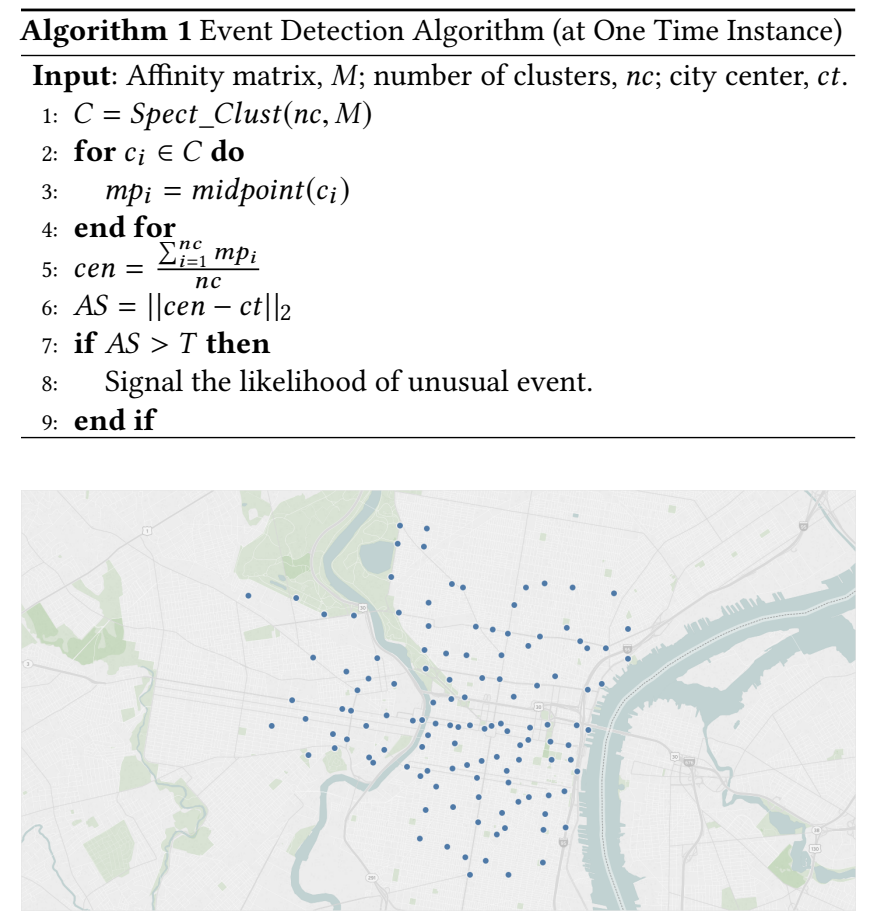

Figure 2: Station Layout of Philadelphia's Indego Bike Sharing System.

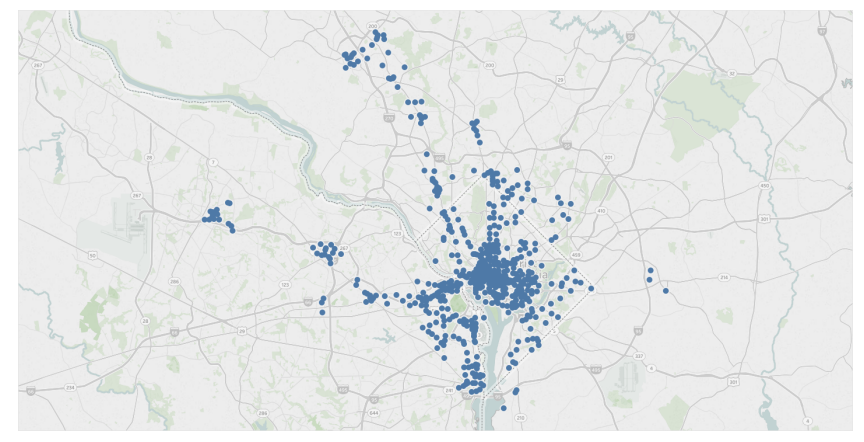

Figure 3: Station Layout of Washington DC's Capital Bike Sharing System.

bikes for citizens to use at their convenience. This system is rather unique because it's reach is across seven different jurisdictions: Washington, DC.; Arlington, VA; Alexandria, VA; Montgomery, MD; Prince George's County, MD; Fairfax County, VA; and the City of Falls Church, VA. Figure 3 shows the station locations for Washongton DC's Capital bike sharing system.

These systems have two main forms of data, Trip History Data (THD) and Real-Time Station Information Data (RSID). The THD is easily accessible from BikeShare system websites as standard located under System Data. This data details specific trip information which we aggregate to build our evolving graphs. In the interest of privacy we only analyze the date, and the start and end location of the trip. RSID however is more difficult to obtain. We set up a server 


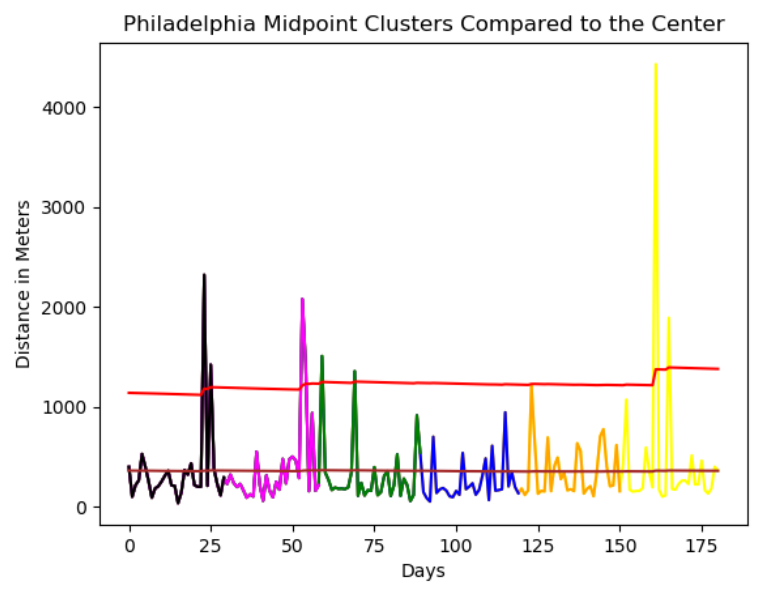

Figure 4: Event Detection for Philadelphia for a 6-month evolving graph time series

with a MongoDB database that every minute would access the API of each BikeShare system we are collecting from and storing the response JSON. Each system has an API for its RSID to accessed, this API follows the General BikeShare Feed Specification (GBFS) as defined by the North American BikeShare Assocation (NABSA). The primary information conveyed by the GBFS is the number of bikes available at a given station at a given time.

After we had conducted our analysis and identified events, we sought to investigate confirmation from real world sources. To investigate what events had occurred at the given time of interest we used news sources, event websites and area college schedules to locate probable events that corresponded to our findings.

\section{EXPERIMENTAL RESULTS}

We present our preliminary results here. First, we analyzed Philadelphia's BikeShare data. Figure 4 shows our detection method identifying eight significant events over a six month period using a threshold of three standard deviations (Upper red line) from the mean (Lower line). Figure 5 shows two examples of 2-cluster representation for Philadelphia during a normal day. The variance of each cluster is spread wider than when there is a single significant local event (see Figure 6, 7, and 8).

Seven of these anomalies (unusual events), we were unable to find insights on, though we believe with better search tools we will be able to. As of now, we see a common trend of these anomalies residing in the same relative area. We also believe that some anomalies may be 'silent' in that they will not be noteworthy events, but instead significant activities at (or near) some stations. Our plan to combat these 'silent' anomalies is to spatially group station regionally. This will reduce single station noise and improve broader crowd flow identification. The clustering on the graph from the first anomaly seen in the time series (in Figure 4) shows that the area with unusual activities is concentrated around Drexel University as shown in Figure 6. However, we were unable to link a known event to the unusual activity.
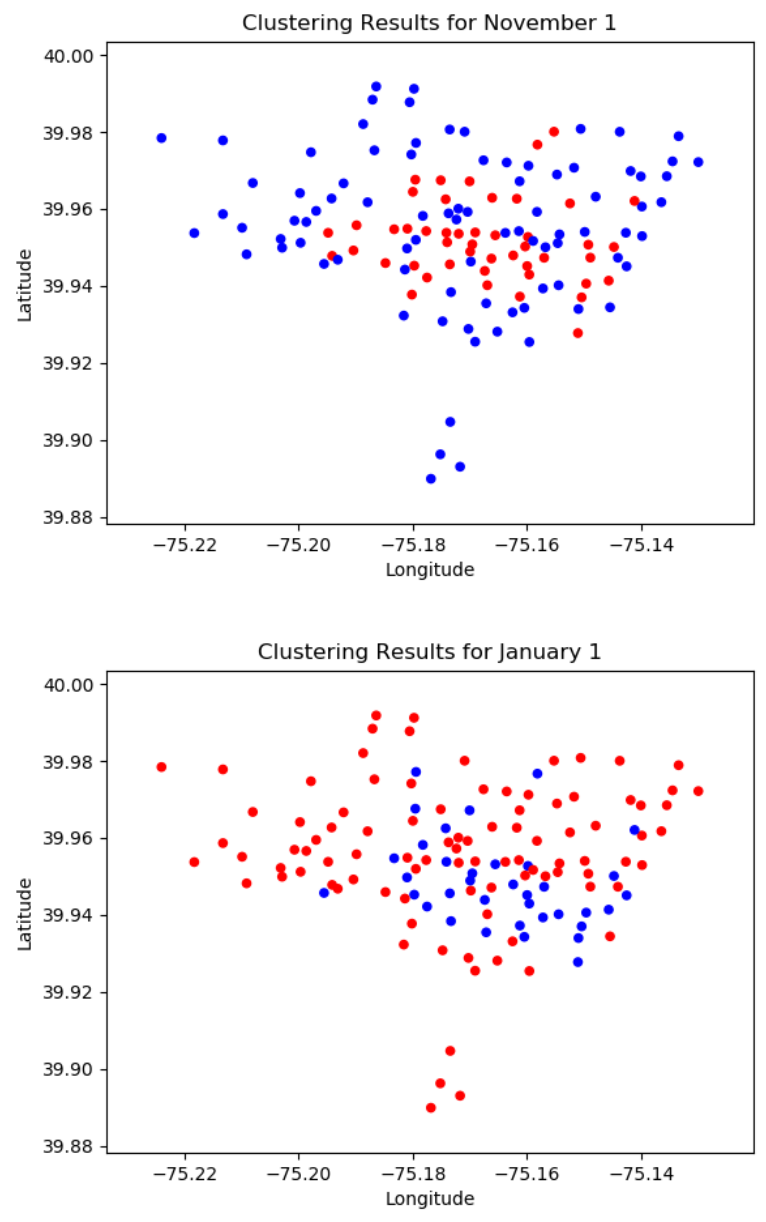

Figure 5: Two examples of normal 2-clusters representation for Philadelphia based on Spectral Clustering.

For the other anomalies that have appeared in our algorithm, they maintain similar positions throughout the range of days. Figure 7 and 8 shows two results of spectral clustering projected to the spatial dimensions for two other unusual events (or activities) near Drexel University. It is interesting that unusual events are detected near the University which could be used to explain and discover student behavior and activities. Also, bike sharing may be a popular mean of transportation for college students in a city.

For Washington DC, our preliminary experimentation fails to find the correct number of clusters to be used for our proposed method to detect unusual events with conclusive evidence or explanation. We experimented with higher number of clusters but unable to find a confirmation of events or explanation for event detection. This could be due to much larger spatial area and more variation in the bike sharing pattern. One potential solution is to partition the Washington DC into smaller regions to detect unusual events. Figure 9, 10, 11 show the event detection algorithm using a threshold of three standard deviations applied to 6-months of 


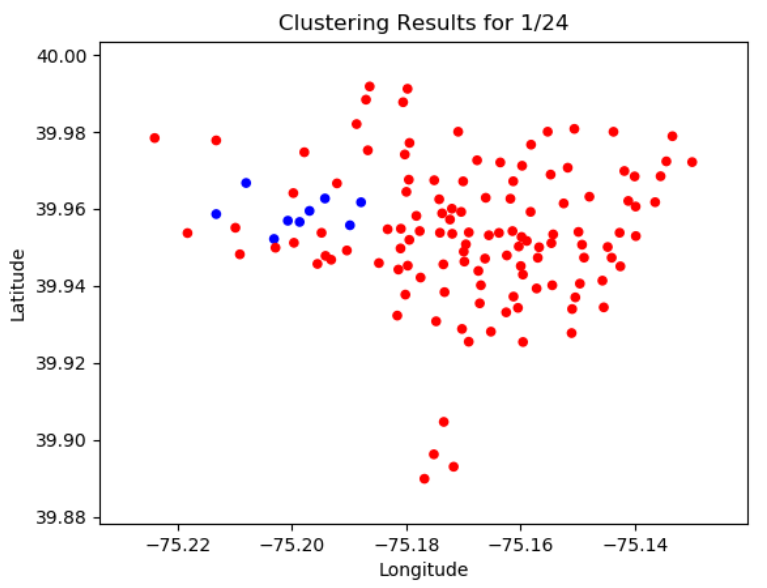

Figure 6: Detecting Unusual Activity Around Drexel University in Philadelphia in January

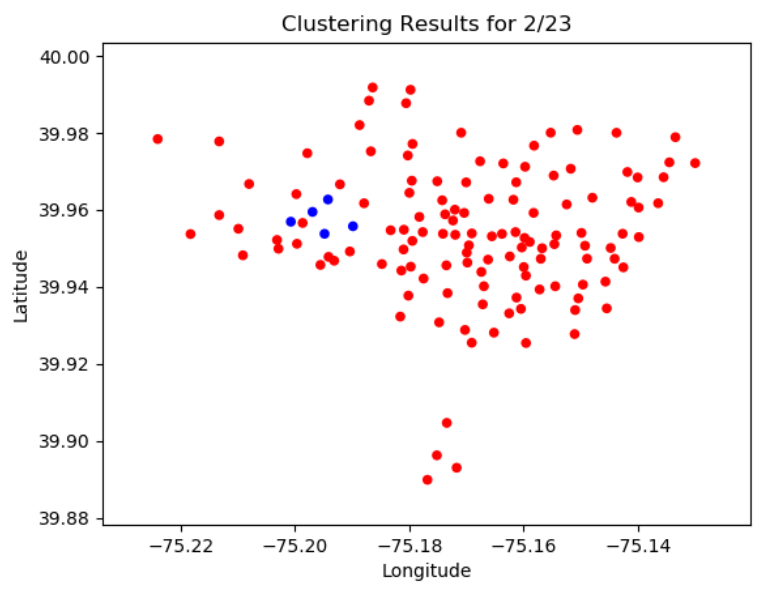

Figure 7: Detecting Unusual Activity Around Drexel University in February

DC dataset as the number of clusters varies from 4 to 6 . The six different colors in the time series represent the six months.

\section{DISCUSSIONS}

In our experimentation we encountered noisy data, infrequently updated data, and difficulties in finding explanations for detected events.

\subsection{Data Issue}

A major issue found in bike sharing systems is that they are not significantly popular in most locations. We collected data from several large cities, but have found most cities' systems do not have enough station activity to be able to rely on their data as an accurate reflection of real world movements and not noise. These low activity stations commonly only see a single digit number of

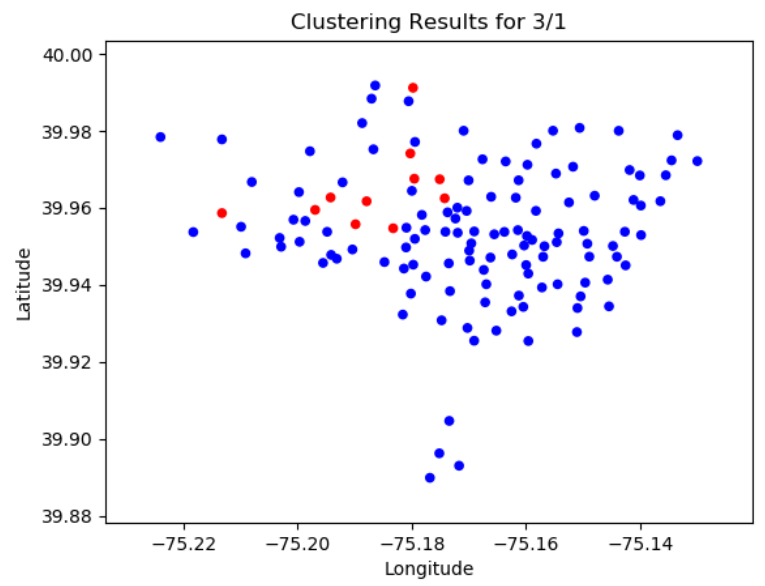

Figure 8: Detecting Unusual Activity Around Drexel University in March

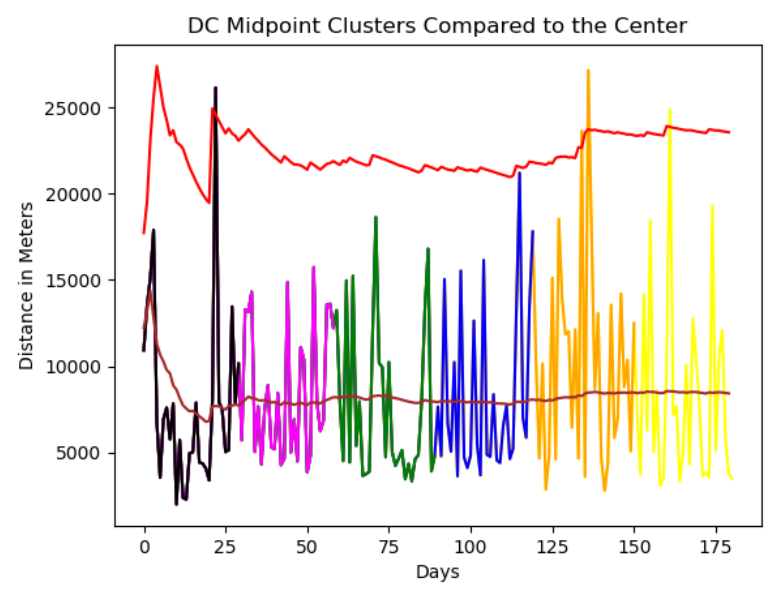

Figure 9: Event Detection for Washington DC for a 6-month evolving graph time series when $n c=4$.

interactions in a day with its busiest stations. We would prefer to see at least a near double digit interaction every day-time hour of a system's busiest stations. A high level of activity is critical to avoid false positives though chance behaviors adding too much noise to analysis and distracting from true anomalies, such as a group of friends spontaneously renting out many bikes.

Bike sharing data for Washington DC and New York Cities provide richer data. However, the spatial extent of two bike share systems are significantly larger. One possible solution is to partition the bike stations into regions and to represent bike flow between regions. Another solution is to partition the bike stations into regions and perform the event detection locally. 


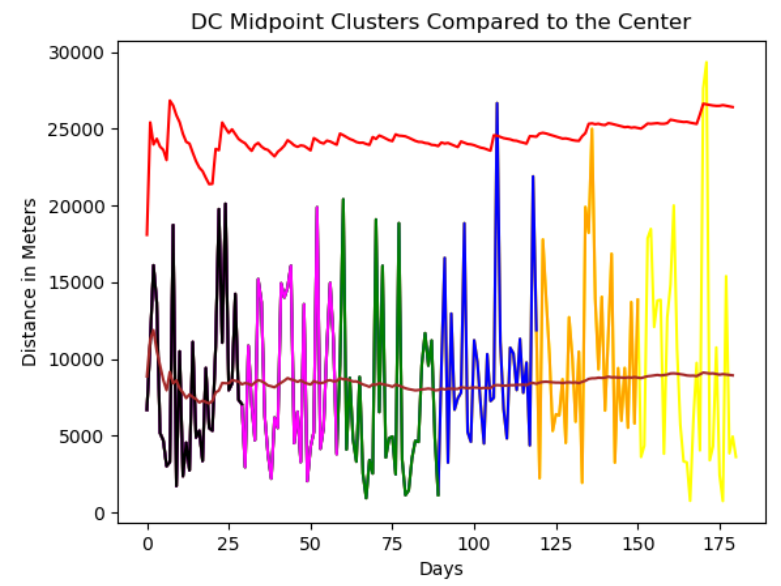

Figure 10: Event Detection for Washington DC for a 6-month evolving graph time series when $n c=5$.

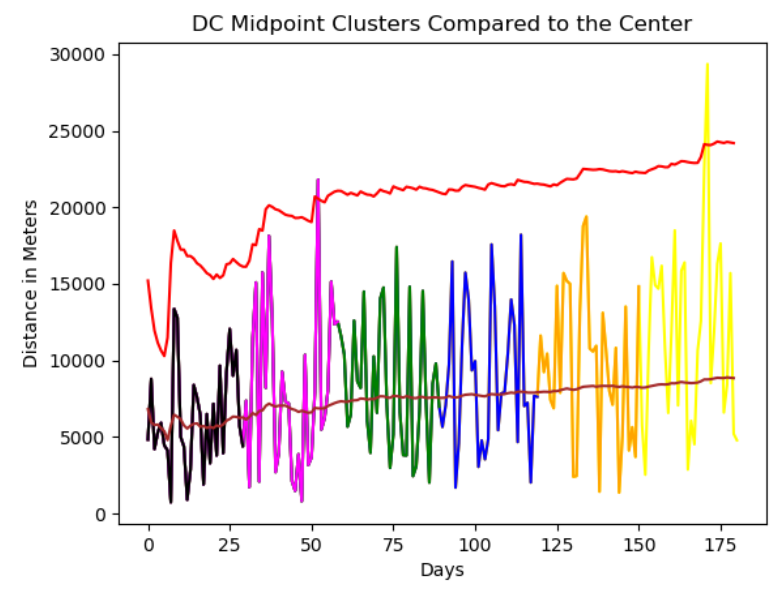

Figure 11: Event Detection for Washington DC for a 6-month evolving graph time series when $n c=6$.

\subsection{Detected Event Explanation}

Another major problem that we encountered was accounting for the detected events/activities. We were often able to detect events in our time series, but we were rarely able to identify the explanation that caused the detection. Due to the real world nature of the problem we cannot be sure if these detection are false or true positives. Perhaps there are new sources that we missed in searching for an explanation or that an unreported event did occur. It may also be the case that our model has a high false positive rate and detected events in fact do not reflect real world events. Moreover, it is challenging to establish ground-truth for proper performance evaluation of the proposed method.

\section{CONCLUSIONS AND FUTURE WORK}

We propose a novel method for the monitoring of usual events/activities in urban environments through the use of publicly accessible bike sharing data, spectral clustering and threshold-based detection. Preliminary results on 6-months of data from Philadelphia and Washington DC are used to show the feasibility of our proposed method.

Currently we struggle to identify the explanation for the detected event. We plan to build an explainable event/activity detection system that not only detects, but explains events. We will link our event detection method with various data sources to enable finding meaning within these events. We plan to (i) increase the number of clusters we analyze as well and (ii) split the bike stations into regional partitions for detection and analysis. This will prove especially useful for larger and more diverse systems such as Washington's Capital BikeShare. We intend to increase the number of cities we analyze to ensure that our method operates on diverse data sets. Further, while our main focus as far as data is to use BikeShare systems we are interested in observing other forms of evolving graph data sets. Lastly, we intend to move our method from a historical analysis to a real-time analysis system.

\section{ACKNOWLEDGMENT}

The research was supported by NSF Award No. 1830489.

\section{REFERENCES}

[1] Shunsuke Aoki, Kaoru Sezaki, Nicholas Jing Yuan, and Xing Xie. 2017. An early event detection technique with bus gps data. In Proceedings of the 25th ACM SIGSPATIAL International Conference on Advances in Geographic Information Systems. ACM, 49.

[2] Farzindar Atefeh and Wael Khreich. 2015. A survey of techniques for event detection in twitter. Computational Intelligence 31, 1 (2015), 132-164.

[3] Deng Cai and Xinlei Chen. 2015. Large scale spectral clustering via landmarkbased sparse representation. IEEE T. Cybernetics 45 (Jan. 2015), 1669-1680.

[4] Eleonora D'Andrea and Francesco Marcelloni. 2017. Detection of traffic congestion and incidents from GPS trace analysis. Expert Systems with Applications 73 (2017), 43-56.

[5] Adrian Dobra, Nathalie E Williams, and Nathan Eagle. 2015. Spatiotemporal detection of unusual human population behavior using mobile phone data. PloS one 10, 3 (2015), e0120449.

[6] Yuxiao Dong, Fabio Pinelli, Yiannis Gkoufas, Zubair Nabi, Francesco Calabrese, and Nitesh V Chawla. 2015. Inferring unusual crowd events from mobile phone call detail records. In foint European conference on machine learning and knowledge discovery in databases. Springer, 474-492.

[7] Tianfu He, Jie Bao, Ruiyuan Li, Sijie Ruan, Yanhua Li, Chao Tian, and Yu Zheng. 2018. Detecting Vehicle Illegal Parking Events using Sharing Bikes' Trajectories.. In KDD. 340-349.

[8] Jianbo Shi and J. Malik. 2000. Normalized cuts and image segmentation. IEEE Transactions on Pattern Analysis and Machine Intelligence 22, 8 (Aug 2000), 888905. https://doi.org/10.1109/34.868688

[9] Siwen Zheng Jiayu Gan, Jianhui Zhang. 2018. Where You Really Are: User Trip Based City Functional Zone Ascertainment. Performance Computing and Communications Conference (IPCCC) 2018 IEEE 37th International (2018), 1-8.

[10] Gilbert Laporte, Frédéric Meunier, and Roberto Wolfler Calvo. 2018. Shared mobility systems: an updated survey. Annals of Operations Research 271, 1 (2018), 105-126.

[11] Rui Li, Kin Hou Lei, Ravi Khadiwala, and Kevin Chen-Chuan Chang. 2012. Tedas: A twitter-based event detection and analysis system. In 2012 IEEE 28th International Conference on Data Engineering. IEEE, 1273-1276.

[12] Lei Lin, Zhengbing He, and Srinivas Peeta. 2018. Predicting station-level hourly demand in a large-scale bike-sharing network: A graph convolutional neural network approach. Transportation Research Part C: Emerging Technologies 97 (2018), 258-276.

[13] Jérémie Jakubowicz Longbiao Chen. 2015. Inferring bike trip patterns from bike sharing system open data. IEEE Big Data (2015), 2898-2900.

[14] Kapileswar Nellore and Gerhard Hancke. 2016. A survey on urban traffic management system using wireless sensor networks. Sensors 16, 2 (2016), 157. 
[15] Eoin O'Mahony and David B Shmoys. 2015. Data analysis and optimization for (citi) bike sharing. In Twenty-ninth AAAI conference on artificial intelligence.

[16] Michael Oppermann, Torsten Möller, and Michael Sedlmair. 2018. Bike Sharing Atlas: Visual Analysis of Bike-Sharing Networks. INTERNATIONAL fOURNAL OF TRANSPORTATION 6, 1 (2018), 1-14.

[17] Bei Pan, Yu Zheng, David Wilkie, and Cyrus Shahabi. 2013. Crowd sensing of traffic anomalies based on human mobility and social media. In Proceedings of the 21st ACM SIGSPATIAL international conference on advances in geographic information systems. ACM, 344-353.

[18] Sugimiyanto Suma, Rashid Mehmood, and Aiiad Albeshri. 2017. Automatic event detection in smart cities using big data analytics. In International Conference on Smart Cities, Infrastructure, Technologies and Applications. Springer, 111-122.

[19] Ulrike Von Luxburg. 2007. A tutorial on spectral clustering. Statistics and computing 17, 4 (2007), 395-416.

[20] Maximilian Walther and Michael Kaisser. 2013. Geo-spatial event detection in the twitter stream. In European conference on information retrieval. Springer, 356-367.

[21] Zuchao Wang, Min Lu, Xiaoru Yuan, Junping Zhang, and Huub Van De Wetering. 2013. Visual traffic jam analysis based on trajectory data. IEEE transactions on visualization and computer graphics 19, 12 (2013), 2159-2168.
[22] Jianshu Weng and Bu-Sung Lee. 2011. Event detection in twitter. In Fifth international AAAI conference on weblogs and social media.

[23] Zidong Yang, Ji Hu, Yuanchao Shu, Peng Cheng, Jiming Chen, and Thomas Moscibroda. 2016. Mobility modeling and prediction in bike-sharing systems. In Proceedings of the 14th Annual International Conference on Mobile Systems, Applications, and Services. ACM, 165-178.

[24] Wangsheng Zhang, Guande Qi, Gang Pan, Hua Lu, Shijian Li, and Zhaohui Wu. 2015. City-scale social event detection and evaluation with taxi traces. ACM Transactions on Intelligent Systems and Technology (TIST) 6, 3 (2015), 40.

[25] Zhenhua Zhang, Qing He, Jing Gao, and Ming Ni. 2018. A deep learning approach for detecting traffic accidents from social media data. Transportation research part C: emerging technologies 86 (2018), 580-596.

[26] Yu Zheng, Huichu Zhang, and Yong Yu. 2015. Detecting collective anomalies from multiple spatio-temporal datasets across different domains. In Proceedings of the 23rd SIGSPATIAL international conference on advances in geographic information systems. ACM, 2.

[27] Xiaolu Zhou. 2015. Understanding spatiotemporal patterns of biking behavior by analyzing massive bike sharing data in Chicago. PloS one 10, 10 (2015), e0137922. 